\title{
THE LOCAL VELOCITY FIELD AND THE HUBBLE CONSTANT
}

\author{
G.A. Tammann \\ Astronomisches Institut der Universität Basel \\ Venusstrasse 7, CH-4102, Binningen, Switzerland \\ and \\ Allan Sandage
}

The Observatories of the Carnegie Institution of Washington

813 Santa Barbara Street, Pasadena CA 91101

"When you reach a fork in the road, take it" (American folklore, sometimes attributed to Yogie Berra).

\section{ABSTRACT}

The methods are reviewed that give a distance modulus to the core of the Virgo cluster of $\mathrm{m}-\mathrm{M}=31.64 \pm 0.08(\mathrm{D}=21.3 \pm 0.8 \mathrm{Mpc})$. It is shown that the cosmic velocity of the cluster core is $1179 \pm 17 \mathrm{~km} \mathrm{~s}^{-1}$, which, when combined with the distance gives $\mathrm{H}_{\mathrm{o}}=55 \pm 3 \mathrm{~km} \mathrm{~s}^{-1} \mathrm{Mpc}^{-1}$ from the Virgo cluster data alone. Nine independent methods are reviewed that confirm that $\mathrm{H}_{\mathrm{o}}=50 \pm 2$. Discussion is made why all methods that are said to give the short distance scale $\left(\mathrm{H}_{\mathrm{o}} \sim 85\right)$ are incorrect.

\section{Introduction}

The debate continues on the value of the Hubble constant despite the multiple evidence from many experiments that its value is near $\mathrm{H}_{\mathrm{o}}=50 \mathrm{~km} \mathrm{~s}^{-1} \mathrm{Mpc}^{-1}$. The purpose of this lecture is to list the evidence for the long distance scale. For those at the fork in the road that wish to still take it, we show why the short distance scale $\left(\mathrm{H}_{\mathrm{O}} \sim 85\right.$ to 100$)$ is not supported by any unbiased data and analysis.

The plan of the report is to (1) show that the local value of $\mathrm{H}_{\mathrm{o}}$ is close to the global value because there is no step in the Hubble diagram that separates the nearby expansion field from the Machian (global) field relative to the microwave background (CMB), (2) show that the global expansion velocity of the Virgo cluster is $\mathrm{v}$ (cosmic) $=1179 \pm 17 \mathrm{~km} \mathrm{~s}^{-1}$ tied to the kinematic frame of the $\mathrm{CMB},(3)$ show that six distance indicators give the distance modulus of the Virgo cluster as $\mathrm{m}-\mathrm{M}=$ $31.64 \pm 0.08(\mathrm{D}=21.3 \pm 0.8 \mathrm{Mpc}),(4)$ show thereby that $\mathrm{H}_{\mathrm{o}}=55 \pm$ 
$2 \mathrm{~km} \mathrm{~s}^{-1} \mathrm{Mpc}^{-1}$ from the Virgo cluster alone, (5) show that the result is confirmed by nine independent methods, the most powerful of which is through distant supernovae of type Ia calibrated in absolute magnitude via Cepheids, (6) justify that the local distance scale from Cepheids is confirmed to within $5 \%$ from the independent calibrations via the old population II objects (RR Lyrae variables, globular clusters, red giant stars), and (7) show how it can be understood that all methods that are said to support the short distance scale $\left(\mathrm{H}_{\mathrm{O}} \sim 85\right)$ are incorrect.

\section{The Local Velocity Field Tied to the CMB}

The two best established motions of the Local Group relative to the Machian frame of the cosmic CMB are (1) the perturbation of the free (cosmic) expansion of the Local group from the Virgo region due to the mass of the Virgo complex, and (2) the larger-scale, nearly bulk motion of the "local bubble" of size $\leq$ $6000 \mathrm{~km} \mathrm{~s}^{-1}$ relative to the $\mathrm{CMB}$, carrying the Local Group and the Virgo complex with it. The model for the salient features is still that set out elsewhere (Tammann \& Sandage 1985), given as Figure 1 here. Refinements on this picture, tying the "local

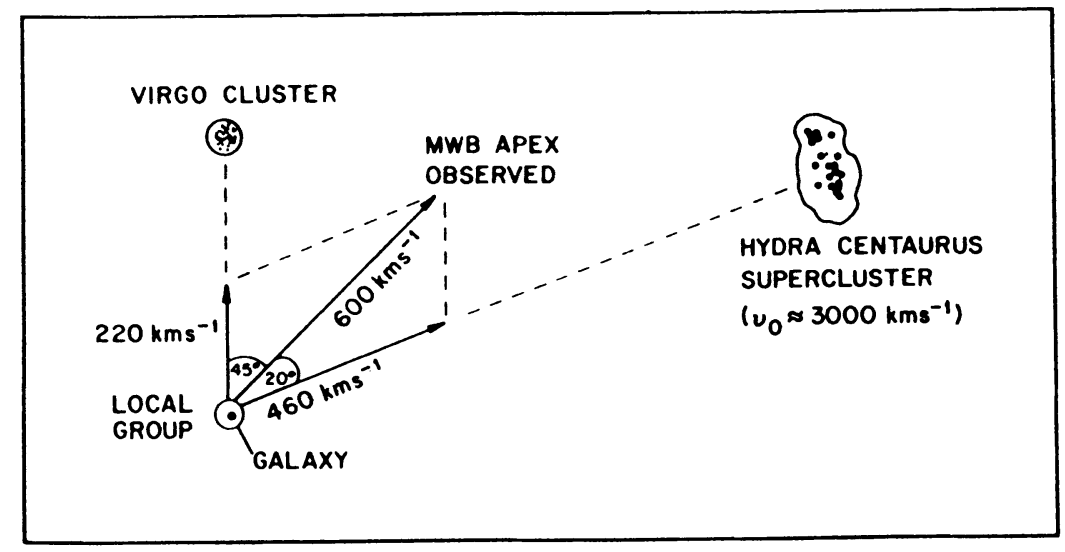

Fig. 1. Vector diagram showing the "infall" (actually retarded expansion) of the Local Group toward the Virgo cluster center plus the supposed motion in the direction of Hydra, which must be caused by the clumpy mass distribution within $\leq 6000 \mathrm{~km} \mathrm{~s}^{-1}$. The two vectors together explain the observed dipole motion toward the warm pole of the CMB. 
bubble" into the Machian cosmic frame can be made using the extensive data of Mathewson, Ford, \& Buchhorn (1992). Analyses by Federspiel et al. (1994, FST) show that the nearly bulk peculiar motion of the local bubble containing the Local Group, the Virgo complex, and the local region gradually peters out beyond $\sim 6000 \mathrm{~km} \mathrm{~s}^{-1}$, merging gradually into the unperturbed Hubble flow. Figures 15 to 19 of FST are decisive on this point.

That the effect of the peculiar dipole motion toward the $\mathrm{CMB}$ within the Local Supercluster and beyond is so small and can be neglected in the determination of $\mathrm{H}_{\mathrm{O}}$ is shown by the lack of a step in the Hubble diagrams at the "edge" of the Local Supercluster. The lack of an effect can be made quantitative. Figure 2 shows the Hubble diagram ( $m$ vs. $\log c z$ ) for nearby clusters and groups to $10,000 \mathrm{~km} \mathrm{~s}^{-1}$ from both hemispheres.

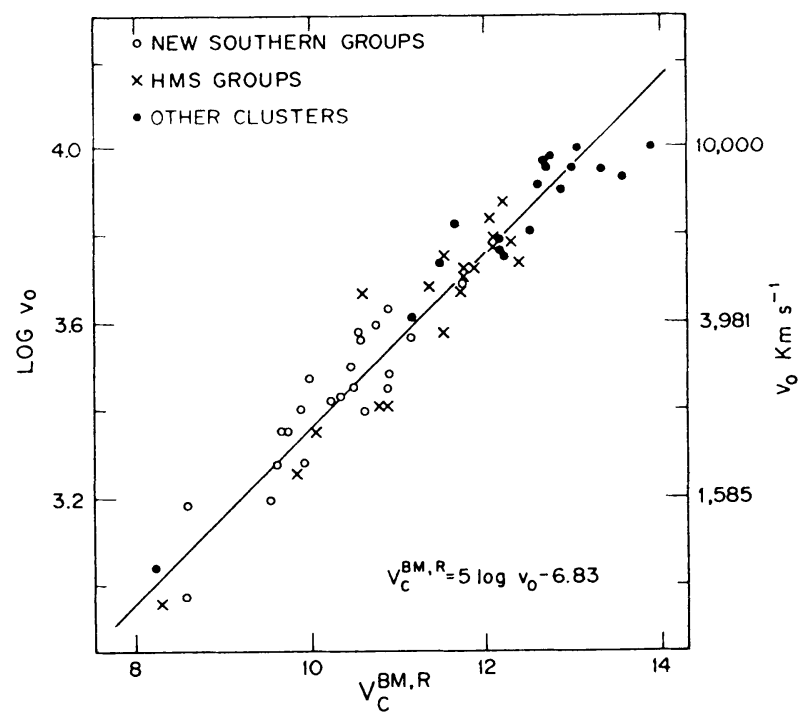

Fig. 2. Hubble diagram for nearby groups and clusters using first-ranked $E$ galaxies corrected for richness and contrast effects. Diagram from Sandage (1975). No steps or large-scale streaming motions are visible.

The data show no step nor any other large-scale streaming motions at the level of more than $\sim 500 \mathrm{~km} \mathrm{~s}^{-1}$. With this limit applied at $5000 \mathrm{~km} \mathrm{~s}^{-1}$, the typical effect of perturbations on the Hubble flow is $<10 \%$. The error on the Hubble constant due to streaming motions will also be equal to or less than this. 
3. The Cosmic Velocity of the Virgo Cluster Core Freed From All Streaming Motions

The most direct method to sample the true expansion field devoid of all effects of local streaming motions is to tie the Virgo cluster core to remote clusters that themselves have known redshifts relative to the kinematic frame of the CMB (Sandage \& Tammann 1990; Jerjen \& Tammann 1993). The result, shown in Figure 3, is that the cosmic (global, Machian) redshift of the

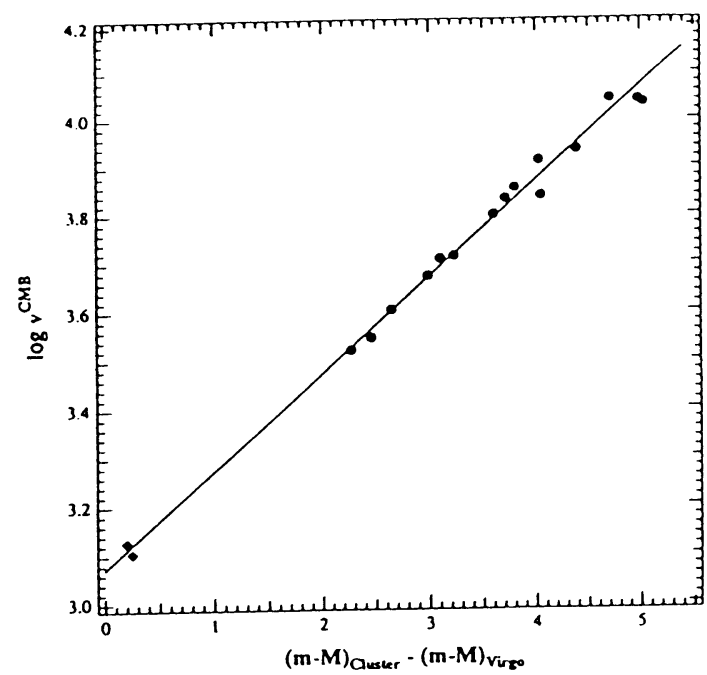

Fig. 3. The Hubble diagram using redshifts in the kinematic frame of the CMB vs. the differences in distance moduli between each of the 17 clusters and the $\mathrm{E}$ galaxies in the Virgo cluster core. The cosmic redshift at the distance of the Virgo cluster core, read from the Hubble line of slope 5 at zero modulus difference, is $1179 \pm 17 \mathrm{~km} \mathrm{~s}^{-1}$.

Virgo cluster core is $\mathrm{v}($ cosmic $)=1179 \pm 17 \mathrm{~km} \mathrm{~s}^{-1}$, devoid of all streaming motions. The result can be used to determine the Hubble constant once the distance to the Virgo cluster is known.

Part of the disagreement in the Hubble constants derived by others is their use of too high a value for v(cosmic) for Virgo. The decisive result from Figure 3 with its error of only $1.4 \%$ removes this one source of the differences between our results and others. 


\section{Six Methods for the Distance to the Virgo Cluster Core}

Before giving the synopses of six methods to find the distance of the Virgo cluster core, we give the summary of the results in Table 1. Results of the two methods (planetary nebulae and surface brightness fluctuations) that are said (Jacoby et al. 1992) to give $(m-M)_{V i r g o}=30.9$, are outside the range of the Table, and are not shown. Methods and data that apparently support the short distance scale are mentioned in section 8 .

\begin{tabular}{|llll|}
\hline TABLE 1 & & & \\
Method & $(\mathrm{m}-\mathrm{M})$ & Galaxy Type & Calibrators \\
Globular clusters & $31.64 \pm 0.25$ & $\mathrm{E}$ & RR Lyraes \\
Novae & $31.57 \pm 0.43$ & $\mathrm{~S}$ & M31, galactic novae \\
Supernovae & $31.63 \pm 0.25$ & E,S & Cepheids, model \\
Dn - $\sigma$ & $31.85 \pm 0.19$ & $\mathrm{E}$ & Galaxy, M31, M81 \\
21 cm line-widths & $31.60 \pm 0.15$ & $\mathrm{~S}$ & 13 nearby calib. \\
Scale length of ScIs & $31.50 \pm 0.20$ & $\mathrm{~S}$ & MW and M31 sizes \\
mean & 31.640 .08 & & $(\mathrm{D}=21.3 \pm 0.08 \mathrm{Mpc})$ \\
\hline
\end{tabular}

(a) Globular clusters: The maximum of the globular cluster luminosity function, calibrated via RR Lyrae distances for MW globular clusters using a new calibration (Sandage 1993c) and via Cepheids for M31 clusters, is applied to the observed luminosity turnover in the cluster samples for five E galaxies associated with the Virgo cluster core (Harris et al. 1991). The modulus first obtained by Harris (1988) of $\mathrm{m}-\mathrm{M}=31.7$ is confirmed (Sandage \& Tammann 1994). The new precepts introduced by Secker \& Harris (1993) that led them to a short distance scale are criticized there (cf. also McLaughlin et al. 1994).

(b) Normal novae: Pritchet \& van den Bergh (1987) discovered nine normal novae in NGC 4472, the brightest $E$ galxay in Virgo subcluster $B$, obtaining a modulus difference between M31 and NGC 4472 of $6.8 \pm 0.4 \mathrm{mag}$. Using a corrected M31 modulus as argued by Sandage \& Tammann (1988) gives (m M) NGC $4472=31.57 \pm 0.43$.

(c) Supernovae: Following Tammann (1988, 1992) and Branch \& Tammann (1992), the Type Ia supernovae observed in Virgo cluster galaxies give $(m-M)=31.54 \pm 0.22$ using a calibration of 
$M_{B}=-19.6$ based on Cepheids (Sandage et al. 1994; Saha et al. 1994b). Schmidt et al. (1992) obtained "expanding envelope" distances for two SNe II in the Virgo cluster of $(\mathrm{m}-\mathrm{M})_{\mathrm{Virgo}}=$ $31.71 \pm 0.26$ mag before they incorrectly (Branch 1994) changed (Schmidt et al. 1994a,b) their value of the dilution (Wagoner) factor. The average of the determinations using $\mathrm{SNe} \mathrm{Ia}$ and $\mathrm{SNe}$ II is shown in the Table.

(d) $D_{n}-\sigma$ : Combining the known variation of surface brightness (SB) of E galaxies with absolute magnitude (Oemler 1974, 1976; Kormendy 1977; Sandage \& Perelmuter 1991) with the Minkowski (1962) relation (later called the Faber-Jackson relation) between absolute magnitude and central velocity dispersion gave a relation between $\mathrm{SB}, \mathrm{M}$, and velocity dispersion (Dressler et al. 1987; Dressler 1987), known now as the $D_{n}-\sigma$ relation. A calibration of the Dressler relation using the bulges of M31, M81 and the MW (Tammann 1988) gives a Virgo modulus of $(\mathrm{m}-\mathrm{M})=31.85 \pm 0.19$.

(e) $21 \mathrm{~cm}$ line widths: Kraan-Korteweg, Cameron, \& Tammann (1988), Fouqué et al. (1990), Teerikorpi (1987), Bottinelli et al. (1987), Sandage (1988a,b, 1994a,b); Federspiel et al. (1994) and undoubtedly others demonstrate that observational selection causes all distances in flux-limited samples to be distorted toward too small values, giving Hubble constants that are too large when using the Tully-Fisher method. Theory and application of the correction for this type of bias (cf. Tammann 1988; KKCT 1988; Fouqué et al. 1990) gives $(\mathrm{m}-\mathrm{M})_{\text {Virgo }}=31.60 \pm 0.15$ for the Virgo cluster, as listed in the Table.

(f) Size of the MW and M31 relative to Virgo spirals: van der Kruit (1986) compares scale lengths of Virgo Sb and Sc galaxies with the known absolute scale lengths of the MW and M31 disks to derive a lower limit to the Virgo cluster distance of 20 $\mathrm{Mpc}$, listed as a real value in the Table.

\section{The Hubble Constant From the Virgo Cluster Distance Itself}

Dividing the cosmic velocity of the Virgo cluster core of $\mathrm{v}=$ $1179 \pm 17 \mathrm{~km} \mathrm{~s}^{-1}$ by the adopted distance from Table 1 gives $\mathrm{H}_{\mathrm{o}}$ (cosmic) $=55 \pm 2 \mathrm{~km} \mathrm{~s}^{-1} \mathrm{Mpc}^{-1}$,

using the Virgo cluster data alone. Six methods that are 
independent of the Virgo cluster method, set out in the next section, also support this long distance scale.

\section{Nine Independent Astronomical and Astrophysical Ways to $\mathrm{H}_{\mathbf{o}}$} The results of nine independent methods to $\mathrm{H}_{\mathrm{o}}$, in addition to the Virgo method, are listed in Table 2. Only the salient literature references are given here. Details of the methods are justified in these references. The principal references are:

\begin{tabular}{|l|l|}
\hline \multicolumn{2}{|c|}{ TABLE 2: Summary of the Various Methods to $\mathrm{H}_{\mathrm{o}}$} \\
Method & $\mathrm{H}_{\mathrm{o}}$ \\
Virgo Distance & $55 \pm 2$ \\
ScI Hubble diagram & $49 \pm 15$ \\
M101 look-alike diameters & $43 \pm 11$ \\
M31 look-alike diameters & $45 \pm 12$ \\
Tully-Fisher field galaxies & $48 \pm 5$ \\
Tully-Fisher cluster data & $55 \pm 8$ \\
Supernovae Ia (B) & $52 \pm 8$ \\
Supernovae Ia (V) & $55 \pm 8$ \\
Supernovae Ia expansion parall. \& ${ }^{56 N i}$ & $50-60$ \\
Sunyaev-Zeldovich effect & $38 \pm 17$ \\
Magn.variations of lensed double QSO & $<70$ \\
mean & $\sim 50$ \\
\hline
\end{tabular}

Virgo distance: last section; ScI galaxy Hubble diagram: The Hubble diagram itself is set out in Sandage \& Tammann (1975). The calibration using the absolute magnitude of the only ScI galaxy with a Cepheid distance (as of 1993), i.e. M101 at $\mathrm{m}-\mathrm{M}=$ 29.3, is in Sandage \& Tammann (1974). The bias properties of the sample and the way to correct for them is in Sandage (1988a); M101 look-alike diameters, calibrated with M101 and corrected for bias is in Sandage (1993a), following the method of van der Kruit (1986); M31 look-alike diameters calibrated by M31 and corrected for bias is discussed in Sandage (1993b); Tully-Fisher field galaxies in the distance-limited sample of KraanKorteweg \& Tammann (1979), calibrated with local galaxies by Richter \& Huchtmeier (1984), give the bias-free result in Table 2 (Sandage 1994b); Tully-Fisher cluster data corrected for the 
"cluster-population-incompletness bias" of Teerikorpi (1987, 1990), is analyzed by Sandage, Federspiel, \& Tammann (1995); The supernovae data for the first two HST calibrations via Cepheids are discussed in Sandage et al. $(1992,1994)$ and Saha et al. (1994a, 1994b). - Purely physical distance determinations are: Type Ia supernovae from $56 \mathrm{Ni}$-powered light curves and expanding-photosphere models (Branch \& Khokhlov 1994); from the Sunyaev-Zeldovich effect (Birkinshaw 1993; Jones 1994); and from gravitational double quasars (Dahle et al. 1994).

\section{The Local Distance Scale is Stable}

The value of $\mathrm{H}_{\mathrm{o}}$ is no better than the reliability of the local distance scale upon which the secondary calibrators rest (involving M31 and all other members of the Local group, used for example by Richter \& Huchtmeier (1984) for the calibration of the TF relation, as well as M81, M101, and others in nearby groups). The reliability of the data for the Local Group is discussed by Tammann (1987, 1992), by Madore \& Freedman (1991), and by Sandage (1995). The agreement on these various distance scales is within a few hundreths of a magnitude in the mean.

The agreement is particularly significant by noting that the distances to four of the local calibrators determined from RR Lyrae stars (LMC, IC 1613, M31, and M33) using the new RR Lyrae calibration given elsewhere (Sandage 1993c) agree with the distances from Cepheids to within less than 0.1 mag (Lee et al. 1993; Tammann \& Sandage 1995).

\section{Criticisms of the Short Distance Scale}

A series of papers have appeared that show how the bias properties of flux-limited samples compared with bias-free distancelimited samples always lead to an incorrect short distance scale and to too large a Hubble constant. Entrance to the literature can be had via Teerikorpi (1987, 1990), Kraan-Korteweg et al. (1988), Sandage (1988a,b; 1994a,b), Bottinelli et al. (1988), Fouqué et al. (1990), Federspiel et al. (1994). All show that proper correction for selection bias reduces the uncorrected Hubble constants near 85 to the range centered near 50 . We do not repeat the arguments here. 
The two remaining methods that are said to give the short distance scale $\left(\mathrm{H}_{\mathrm{o}}=85\right)$ are the planetary nebulae (PNe) and the surface brightness fluctuation method (SBFM). It is useful to mention our reservations about them here.

The PN method relies on a cutoff of their luminosity function in the $\lambda 5007 \AA$ light. PNe do not have an infinitely sharp luminosity function at the bright end. Therefore the method is susceptable to population bias. Large samples will have brighter first-ranked PNe than small samples. The nine brightest PNe in Jacoby's et al. (1990) sample are brighter than the vertical "asymptote" of the luminosity function finally adopted in Jacoby et al. (1992). The consequences of the nonsharp bright end to the LF are discussed by Bottinnelli et al. (1991), by Tammann (1993), and also by Méndez et al. (1993).

Comparison of relative distances determined by the SBFM and the $D_{n}-\sigma$ method show that the former are smaller by an average of $25 \%$ (Tammann \& Sandage 1995). More seriously, the individual distances for $13 \mathrm{E}$ and S0 galaxies in the Virgo cluster (Tonry, Ajhar, \& Luppino 1990) show a large scatter in their individual distances (12 to $23 \mathrm{Mpc}$ ), but also that these distances are strongly correlated with metallicity (Tammann 1992), indicating uncorrected systematics in the method itself. Consequently Tonry (1991) introduced the V - I color as an additional free parameter, but some metallicity dependence yet remains (Lorenz et al. 1993), with the faint, metal poor ones being (artificially) nearer. In addition, in the one case where a direct comparison with a Cepheid distance is possible (NGC 5253), the SBFM gives $2.5 \mathrm{Mpc}$ whereas the Cepheid distance is $3.9 \mathrm{Mpc}$, i.e. a distance ratio of a factor of 1.6.

Finally, it is necessary to record our disbelief concerning the announcement of the solution to $\mathrm{H}_{\mathrm{o}}$ by Pierce et al. (1994), which also is the communication by van den Bergh in this volume. Our concerns center on (1) the technical aspects of their data, and their displayed P-L relation, and (2) their precept that the distance, even if correct, of the one spiral that is the most easily resolved in the total spiral sample of Virgo "associates" has a connection to the distance of the E galaxy Virgo core. 
Support of the Swiss National Sci. Foundation is gratefully acknowledged.

\section{REFERENCES}

Birkinshaw, M. 1993, Harvard-Smithsonian Center of Ap., preprint no. 3725.

Bottinelli, L., Fouqué, P., Gouguenheim, L., Paturel, G., \& Teerikorpi, P. 1987, A\&A, 181, 1

Bottinelli, L., Gouguenheim, L., Paturel, G., \& Teerikorpi, P. 1988, ApJ, 328, 4

Bottinnelli, L., Gouguenheim, L., Paturel, G., \& Teerikorpi, P. 1991, A\&A, 252, 550

Branch, D. 1995, Proc. Seventh Marcel Grossman Conf., eds. M. Kaiser, \& R. Jantzen (World Scientific), in press

Branch, D., \& Khokhlov, A.M. 1994, Physics Reports, in press

Branch, D. \& Tammann, G.A. 1992 Ann. Rev. A\&A, 30, 359

Dahle, H., Maddox, S.J., \& Lilje, P.B. 1994, ApJ Letters, in press

Dressler, A. 1987, ApJ, 317, 1

Dressler, A. et al. 1987, ApJ, 313, 42

Feast, M. 1994, MNRAS, 266, 255

Federspiel, M., Sandage, A., \& Tammann, G.A. 1994, ApJ, 430, 29

Fouqué, P., Bottinelli, L., Gougueheim, L., \& Paturel, G. 1990, ApJ, 349, 1

Harris, W.E. 1988, in The Extragalactic Distance Scale, ed. S. van den Bergh \& C.J. Pritchet, ASP Conf. Ser. Vol. 4, p 231

Harris, W.E., Allwright, J.W.B., Pritchet, C.J., \& van den Bergh, S. 1991, ApJS, 76, 115

Jacoby, G., Ciardullo, R., \& Ford, H.C. 1990, ApJ, 356, 332

Jacoby, G. et al. 1992, PASP, 104, 599

Jerjen, H., \& Tammann, G.A. 1993, A\&A, 276, 1

Jones, M. 1994, Ap.Letters \& Comm., in press

Kormendy, J. 1977, ApJ, 218, 333

Kraan-Korteweg, R.C., Cameron, L.M., \& Tammann, G.A. 1988, ApJ, 331, 620

Kraan-Korteweg, R.C.\& Tammann, G.A. 1979, Astron. Nach., 300, 181

Lee, M.G., Freedman, W.L., \& Madore, B.F. 1993, ApJ, 417,

Lorenz, H., Böhm, P., Capaccioli, M., Richter, G.M., \& Longo, G. 1993, A\&A, 277, L15

Madore, B.F. \& Freedman, W.L. 1991, PASP, 103, 933

Mathewson, D.S., Ford, V.L., \& Buchhorn, M. 1992, ApJS, 81, 413

McLaughlin, D.E., Harris, W.E., \& Hanes, D.A. 1994, ApJ 422, 486

Méndez, R.H., Kudritzki, R.P., Ciardullo, R., \& Jacoby, G.H. 1993, A\&A 275, 534

Minkowski, R. 1962, in Problems in Extragalactic Research, IAU Symp. 15, ed. G.C. McVittie, (Macmillan Co.), p 112

Oemler, A. 1974, ApJ, 194, 1

Oemler, A. 1976, ApJ, 209, 693

Pierce, M. et al. 1994, Nature, 371, 385 
Pritchet, C.J., \& van den Bergh, S. 1987, ApJ, 318, 507

Richter, O.-G., \& Huchtmeier, W.K. 1984, A\&A, 132, 253

Saha, A. et al. 1994a, ApJ, 425, 14 1994b, ApJ, in press

Sandage, A. 1975, ApJ, 202, 563

--.- 1988a, ApJ, 331, 583

-- 1988b, ApJ, 331, 605

1993a, ApJ, 402, 3

1993b, ApJ, 404, 419

1993c, AJ, 106, 703

1994a, ApJ, 430, 1

1994b, ApJ, 430, 13

1995, the 23rd Sass Fee Course of the Swiss Astron. Soc., eds. B.

Binggeli \& R. Buser (Springer Verlag), in press

Sandage, A., Federspiel, M., \& Tammann, G.A. 1995, ApJ submitted

Sandage, A. \& Perelmuter, J.-M. 1991, ApJ, 370, 455

Sandage A., \& Tammann, G.A. 1974, ApJ, 190, 525

1975, ApJ, 197, 265

1988, ApJ, 328, 1

1990, ApJ, 365, 1

1994, ApJ, in press

Sandage, A., Saha A., Tammann, G.A., Panagia, N., \& Macchetto, D. 1992, ApJ, 401, L7

Sandage, A., Tammann, G.A., Labhardt, L., Schwengeler, H., Panagia, N., \& Macchetto, F.D. 1994, ApJ, 423, L13

Schmidt, B.P., Kirshner, R.P., \& Eastman, R.G. 1992, ApJ, 395,

Schmidt, B.P. et al. 1994, AJ, 107, 1444

Secker, J., \& Harris, W.E. 1993, AJ, 105, 1358

Tammann, G.A. 1987, in Relativistic Astrophysics, ed. M. Ulmer, 13th Texas Symp. World Scientific (Singapore), p. 8

1988, in The Extragalactic Distance Scale, ed. S. van den

Bergh, \& C.J. Pritchet, ASP. Conf. Ser. No. 4, p 282,

1992, Physica Scripta, T43, 31

1993, in Planetary Nebulae, IAU Symp. 155, eds. R. Wein-

berger, \& A. Acker (Dordrecht: Kluwer), p 515

Tammann, G.A. \& Sandage, A. 1985, ApJ, 294, 81 1995, Lectures at the 3rd International School "D.

Chalonge", Erice, Nov. 1994, in press

Teerikorpi, P. 1987, A\&A, 173, 39

- 1990, A\&A, 234, 1

Tonry, J.L. 1991, ApJ, 373, L1

Tonry, J.L., Ajhar, A.E., \& Luppino, G.A. 1990, AJ, 100, 1416

van der Kruit, P. 1986, A\&A, 157, 230 